\title{
Silencing the nosocomial pathogen Serratia marcescens by glyceryl trinitrate
}

\author{
Hisham A Abbas ${ }^{1}$, Ahmed M Elsherbini ${ }^{2}$
}

1. Department of Microbiology and Immunology, Faculty of Pharmacy, Zagazig University- Zagazig- Egypt.

2. Health Sciences College-Umm Al Qura University, AlQunfudah, Saudi Arabia.

\begin{abstract}
Background: Quorum sensing is a cell-to-cell communication system in bacteria that controls the production of virulence factors. Serratia marcescens is a causative agent of hospital-acquired infections that shows high resistance to antibiotics. This makes the treatment of these infections difficult. Quorum sensing regulates the production of virulence factors of $S$. marcescens such as prodigiosin, protease, swimming and swarming motilities and formation of biofilms. Inhibition of quorum sensing may be an alternative to antibiotic treatment to avoid emergence of resistance.

Objectives: Testing the ability of glyceryl trinitrate to inhibit quorum sensing and virulence factors of Serratia marcescens.

Methods: The inhibiting activities of sub-inhibitory concentration of glyceryl trinitrate against the quorum-sensing regulated violacein pigment in Chromobacterium violaceum CV026 was performed to evaluate the anti-quorum sensing effect of glyceryl trinitrate. The anti-virulence activity was assessed against prodigiosin, protease, biofilm formation in addition to swimming and swarming motilities.

Results: Glyceryl trinitrate at at a concentration of $0.25 \mathrm{mg} / \mathrm{ml}$ produced significant inhibitory effects against violacein (67.01\%), prodigiosin (82.67\%), protease, swimming (36.72\%) and swarming $(79.31 \%)$ motilities and biofilm formation (87.83\%).

Conclusion: Glyceryl trinitrate is a quorum sensing and virulence inhibitor that may be useful in treatment of nosocomial infections caused by Serratia marcescens.

Keywords: Serratia marcescens, quorum sensing, virulence, glyceryl trinitrate.

DOI: https://dx.doi.org/10.4314/ahs.v18i1.2

Cite as: Abbas HA, Elsherbini AM. Silencing the noscomial pathogen Serratia marscescens by glyceryl trinitrate. Afri Health Sci 2018;18(1): 1-10. bttps://dx.doi.org/10.4314/abs.v18i1.2
\end{abstract}

\section{Introduction}

Serratia marcescens is a nosocomial pathogen that can lead to many nosocomial infections such as those affecting urinary tract, respiratory tract in addition to wound in-

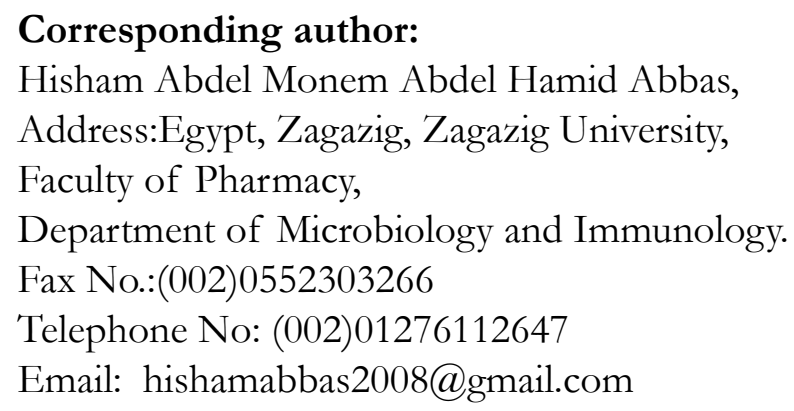

fections. It has an arsenal of virulence factors that aid in escape from the immune system and causing these infections such as prodigiosin, proteases, biofilm and its ability to swim and $\operatorname{swarm}^{1-3}$. These virulence factors are controlled by a mechanism of intercellular communication termed quorum sensing. Quorum sensing enables bacterial cells to monitor their numbers and respond by coordinating the expression of genes including virulence genes $^{2-4}$. Some strains of Serratia marcescens cause infections that are hard to treat. The underlying reason is their high resistance to many categories of antibiotics involving fluoquinolones, aminoglycosides and $\beta$-lactams ${ }^{5,6}$. The high virulence and resistance of Serratia marcescens necessitates seeking a new treatment strategy. It is supposed 
that quorum sensing inhibition is one useful approach that can help in treating infections without exerting stress on the growth of bacteria to avoid the evolution of antibiotic resistance. Furthermore, it can enhance the immune clearance of the pathogens ${ }^{7,8}$.

Glyceryl trinitrate (GTN) is an anti-hypertensive that has anti-microbial properties ${ }^{9,10}$. It could inhibit the growth of Candida albicans and Pseudomonas aeruginosa planktonic growth $^{10,11}$. Moreover, GTN exerted antibiofilm activity against Staphylococcus aureus, Staphylococcus epidermidis, Pseudomonas aeruginosa and candida albicans ${ }^{12}$. In our previous study, GTN was found to inhibit quorum sensing in Pseudomonas aeruginosa ${ }^{11}$. GTN is an FDA approved drug that can be used topically for anal fissures at concentrations that can reach $0.4 \%{ }^{13}$.

This study aimed to investigate the potential anti-quorum sensing and anti-virulence activities of GTN against the nosocomial pathogen Serratia marcescens.

\section{Materials and methods}

\section{Media and chemicals}

Tryptone soya broth, Mueller Hinton broth and Mueller Hinton agar were the products of Oxoid (Hampshire, UK). Luria-Bertani (LB) broth and LB agar were purchased from Lab M Limited (Lancashire, United Kingdom).Glyceryl trinitrate was obtained from POHLBoskamp, Gmbh\&Co., Hohenlockstedt, Germany (Stock solution of $1 \mathrm{mg} / \mathrm{ml})$. Other chemicals were of pharmaceutical grade.

\section{Bacterial Strains}

The Serratia marcescens isolate in this study was used in a previous study ${ }^{14}$. It is a clinical one obtained from an Intensive Care Unit patient admitted to Zagazig University Hospital by endotracheal aspiration. The MALDI-TOFF apparatus at the Clinical Pathology Department, Faculty of Medicine, Zagazig University was used for identification of this isolate. The biosensor strain Chromobacterium violaceum CV026 was obtained from the Department of Microbiology, Faculty of Pharmacy, Ain Shams University.

\section{Determination of Minimum Inhibitory Concentra- tion (MIC)}

The agar dilution method was used in determination of the minimum inhibitory concentration of GTN ac- cording to the Clinical Laboratory and Standards Institute Guidelines (CLSI) ${ }^{15}$. The tested strain was incubated overnight in tryptone soya broth (TSB) and the suspension was diluted with Mueller-Hinton broth in order to prepare a suspension with a turbidity approximating that of $0.5 \mathrm{McF}$ arland Standard. The suspension was further diluted with sterile saline $(1: 10)$. By using a micropipette, a standardized inoculum (approximately $10^{4} \mathrm{CFU}$ per spot) was spotted on the surface of Mueller-Hinton agar plates containing different GTN concentrations and control plate without GTN. The MIC of GTN was the lowest concentration that inhibits growth on the plate after incubation at $37^{\circ} \mathrm{C}$ for $20 \mathrm{~h}$.

\section{Effect of GTN on bacterial growth}

The effect of sub-inhibitory concentration of GTN on the growth of the tested strain of $S$. marcescens was detected according to Nalca et al. ${ }^{16}$ overnight culture from $S$. marcescens was prepared in LB broth and adjusted to 0.5 McFarland Standard. The prepared suspension was used to inoculate LB broth containing $0.25 \mathrm{mg} / \mathrm{ml}$ of GTN and control LB broth without GTN so that the final inoculum is approximately $1 \mathrm{X} 10^{6} \mathrm{CFU} / \mathrm{ml}$. After overnight incubation at $37^{\circ} \mathrm{C}$, the optical densities of both cultures were measured at $600 \mathrm{~nm}$ by using Biotek Spectrofluorimeter (Biotek, USA).

\section{Violacein inhibition assay}

To determine the quorum sensing inhibiting activity of GTN, the biosensor strain Chromobacterium violaceum CV026 was used according to Choo et $\mathrm{al}^{17}$. A suspension of the tested strain with $\mathrm{OD}_{600}$ of 1 was prepared from overnight culture. Aliquots of $100 \mu$ l of bacterial suspension were added to the wells of a 96-well microtiter plate to which aliquots of $100 \mu \mathrm{l}$ of LB broth with N-hexanoyl homoserinelactone in the presence and absence of 0.25 $\mathrm{mg} / \mathrm{ml}$ of GTN were delivered. The plate was incubated at $28^{\circ} \mathrm{C}$ for $16 \mathrm{~h}$ and was dried at $60^{\circ} \mathrm{C}$. The purple pigment violacein was extracted by addition of aliquots of 100 $\mu \mathrm{l}$ of DMSO to the wells and incubation at $30^{\circ} \mathrm{C}$ with shaking. DMSO was used as negative control and the absorbance was measured at $590 \mathrm{~nm}$ using Biotek Spectrofluorimeter (Biotek,USA).

\section{Biofilm inhibition assay}

The tested strain was reported as a strong biofilm form- 
ing isolate ${ }^{14}$. To determine the ability of GTN to inhibit biofilm formation, the modified method of Abraham et al. ${ }^{18}$ was used. A suspension of $S$. marcescens strain was prepared from overnight culture in tryptone soya broth (TSB) and its optical density was adjusted to $\mathrm{OD}_{600}$ of $0.4\left(1 \times 10^{8} \mathrm{CFU} / \mathrm{ml}\right)$ was added. Aliquot of $10 \mu \mathrm{l}$ of the suspension was added to $1 \mathrm{ml}$ amounts of fresh TSB with and without $0.25 \mathrm{mg} / \mathrm{ml}$ of GTN. Aliquots of $100 \mu \mathrm{l}$ of TSB with and without GTN were delivered into the wells of 96 wells microtiter plate and incubated at $28^{\circ} \mathrm{C}$ for 24 $\mathrm{h}$. The planktonic cells were aspirated and the wells were washed three times with distilled water and left to dry. The attached cells were fixed with methanol for 20 minutes and stained with crystal violet $(1 \%)$ for 20 minutes. The wells were washed and the attached dye was eluted by $33 \%$ glacial acetic acid. The absorbance was measured at $590 \mathrm{~nm}$ using Biotek Spectrofluorimeter (Biotek, USA). The percentage of biofilm inhibition was calculated using the following formula

$\%$ of biofilm inhibition $=\left[\mathrm{OD}_{600}\right.$ control- $\mathrm{OD}_{600}$ in presence of ambroxol]/ $\mathrm{OD}_{600}$ control

\section{Microscopic visualization of biofilm inhibition by the light microscope and scanning electron micro- scope}

In order to analyze biofilm inhibition, the method of Sakar et al. ${ }^{19}$ was followed with some modification. The biofilm of the tested strain of Serratia marcescens was formed on glass cover slips placed in polystyrene petri plates in the presence and absence of $0.25 \mathrm{mg} / \mathrm{ml}$ of GTN. The plates were incubated for $24 \mathrm{~h}$ at $28^{\circ} \mathrm{C}$, the cover slips were washed with water three times and stained with crystal violet (1\%) for 20 minutes. The cover slips were examined after staining under the light microscope at a 400X magnification. For scanning electron microscopic (SEM) analysis, biofilms formed on glass cover slips were fixed by glutaraldehyde $(2.5 \%)$ for 120 minutes. After washing with distilled water, the cover slips were dehydrated by increasing concentrations of ethanol $(50 \%, 60 \%, 70 \%$, $80 \%, 90 \%$ and $100 \%$ ) for 30 seconds. The samples were gold coated after critical-point drying and examined under scanning electron microscope $e^{20}$.

\section{Swimming and swarming motilities assay}

The ability of GTN to block the swimming and swarming motilities was detected according to Matsuyama et al. ${ }^{21}$ For swimming assay, LB agar plates containing $0.3 \%$ agar with and without $0.25 \mathrm{mg} / \mathrm{ml}$ GTN were prepared. Overnight culture of S.marcescens in LB broth was prepared and $5 \mu \mathrm{l}$ of the suspension was inoculated into the center of the plates. Swarming LB gar plates with $0.5 \%$ agar containing $0.25 \% \mathrm{mg} / \mathrm{ml}$ of GTN and control plates were point inoculated with $5 \mu$ l of the prepared suspension. The plates were incubated at $28^{\circ} \mathrm{C}$ for $20 \mathrm{~h}$. The zones of swimming and swarming were measured. The experiment was made in triplicates and the results were averaged.

\section{Prodigiosin inhibition assay}

The production of prodigiosin by $S$. marcescens was quantified in the presence and absence of GTN. The tested strain was grown overnight in LB broth and adjusted to $\mathrm{OD}_{600}$ of 0.4 and inoculated in $2 \mathrm{ml}$ fresh LB broth and incubated at $28^{\circ} \mathrm{C}$ for $18 \mathrm{~h}$. The cells were collected by centrifugation at $13000 \mathrm{rpm}$ for 10 minutes. To extract prodigiosin, acidified ethanol $(4 \% 1 \mathrm{M} \mathrm{HCl}$ in ethanol) was used. The absorbance was measured at $534 \mathrm{~nm}$ using Biotek Spectrofluorimeter (Biotek, USA) and the degree of inhibition was determined. The experiment was made in triplicate and the results were averaged ${ }^{22}$.

\section{Protease assay}

To assay the inhibitory activity of GTN on protease production, the qualitative skim milk agar method was used ${ }^{23}$. Skim milk LB agar plates (5\% skim milk) with and without GTN $(0.25 \mathrm{mg} / \mathrm{ml})$ were prepared. The plates were surface inoculated, each with $5 \mu$ of overnight culture of S. marcescens in $\mathrm{LB}$ broth and incubated at $28^{\circ} \mathrm{C}$ for $20 \mathrm{~h}$. The diameter of the clear zones surrounding the growth were measured.

\section{Statistical analysis}

The inhibitory activities of GTN on quorum sensing and virulence factors of Serratia marcescens were compared by $t$ test, Graph Pad Prism 5. P values $<0.05$ were considered statistically significant.

\section{Results}

\section{Determination of MIC}

Glyceryl trinitrate could not inhibit the growth of Serratia marcescens isolate at $0.5 \mathrm{mg} / \mathrm{ml}$ which is the maximum available concentration (stock solution of GTN is of 
concentration of $1 \mathrm{mg} / \mathrm{ml}$ ), so MIC is equal to or higher than $1 \mathrm{mg} / \mathrm{ml}$. the concentration selected to test the anti-quorum sensing and anti-virulence activities of GTN is $0.25 \mathrm{mg} / \mathrm{ml}$ which is equivalent to $1 / 4 \mathrm{MIC}$ or less.
Determination of the effect of GTN on bacterial growth

To determine the effect of GTN on growth, the optical densities of the bacterial suspension at $600 \mathrm{~nm}$ following overnight incubation in LB broth were measured in the presence or absence of GTN. No statistically significant difference was found between the turbidities of the bacterial suspension with or without GTN indicating the lack of the effect of GTN on growth (Fig. 1).

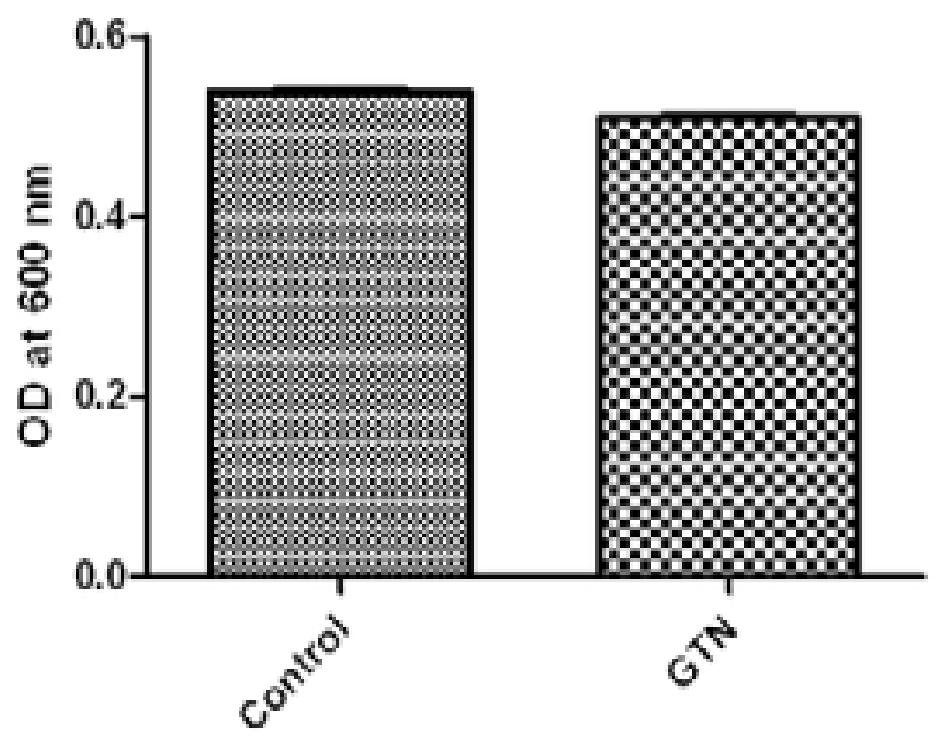

Figure 1. Effect of GTN on growth of Serratia marcescens. No statistically significant difference between OD $_{600}$ of the GTN treated and untreated cultures after overnight incubation in LB broth.

\section{Assessment of biofilm inhibition}

The biofilm formation was quantified to show the ability of GTN to interfere with biofilm production. GTN at
$0.25 \mathrm{mg} / \mathrm{ml}$ produced statistically significant reduction in biofilm biomass $(p<0.05)$. The percentage of biofilm inhibition reached $87.83 \%$ (Fig. 2).

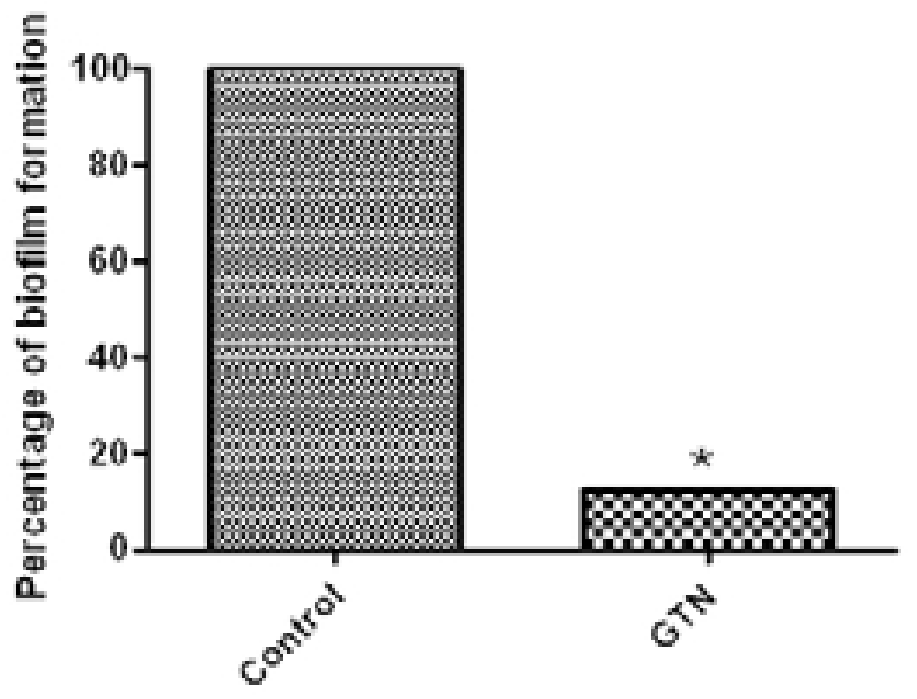

Figure 2. Biofilm inhibition of $S$. marcescens by GTN.*, significant $P<0.05$. 
To further explore the biofilm inhibiting activity of GTN, light microscopic examination of biofilms formed on glass cover slips in the presence and absence of GTN was performed. In the presence of GTN, both the thickness and surface coverage were markedly decreased (Fig.
3). Furthermore, the SEM images clearly illustrated the biofilm inhibiting activity of GTN. In GTN treated sample, very few scattered cells were observed as compared to the untreated sample that showed the complex biofilm structure with aggregated cells.

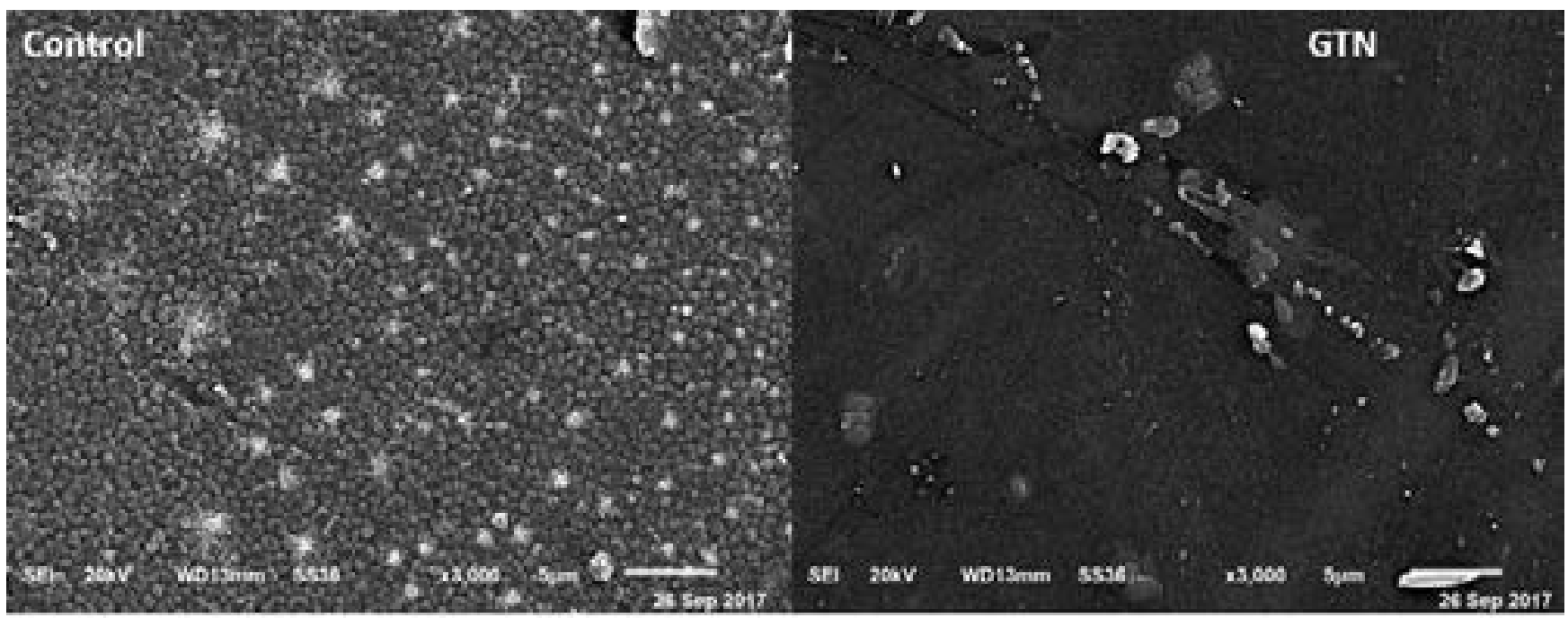

a
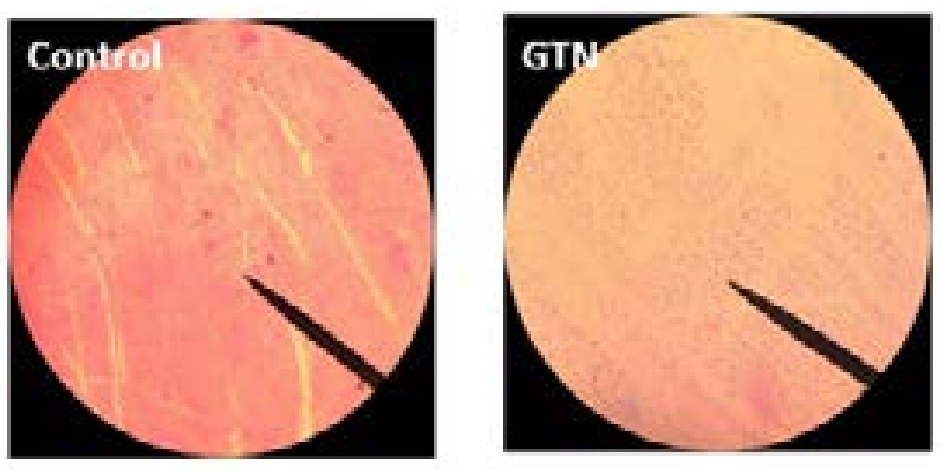

b

Figure 3. Microscopic analysis of biofilm inhibition by GTN (a) SEM images and (b) Light microscopic images of GTN treated and untreated biofilms.

\section{Inhibition of violacein production}

To prove the quorum sensing inhibition by GTN, the production of violacein pigment in the biosensor strain Chro- mobacterium violaceum CV026 that is controlled by quorum sensing mechanism was monitored. GTN significantly diminished violacein production (67.01\%). This confirms that GTN is a quorum sensing inhibitor (Fig. 4). 


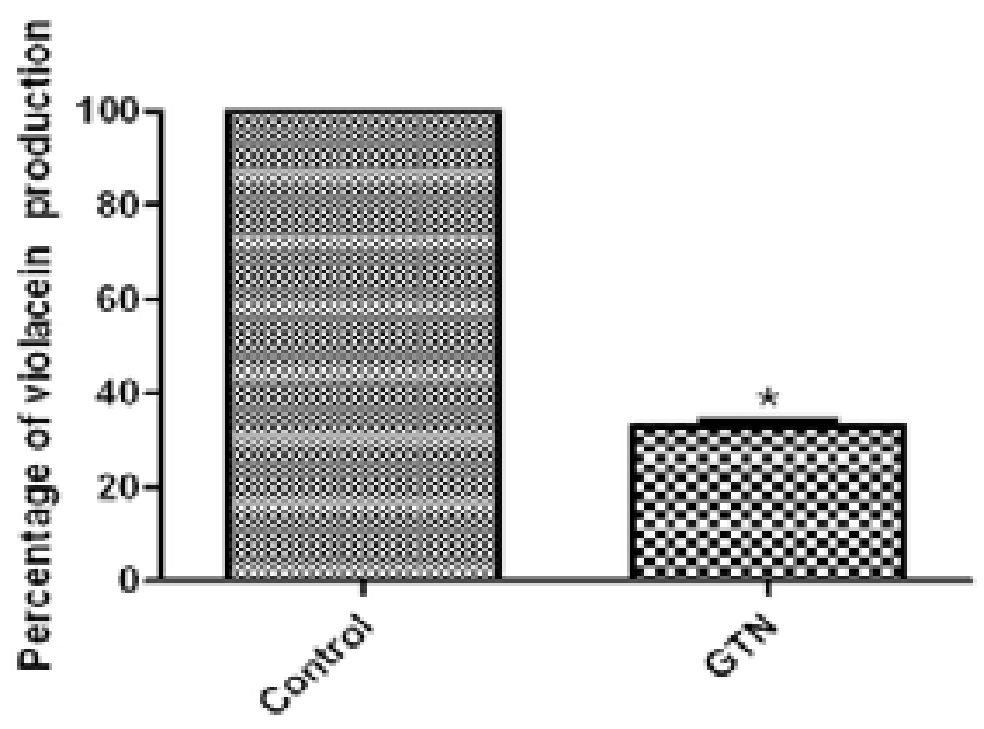

Figure 4. Inhibition of violacein pigment of Chromobacterium violaceum CV026 by GTN. *, significant $\mathrm{P}<0.05$.

Prodigiosin inhibition assay

Prodigiosin is another quorum-sensing controlled pigment that is produced by $S$. marcescens. GTN showed a significant ability to inhibit prodigiosin production. The inhibition percentage achieved was $82.67 \%$ (Fig 5).

Inhibition of Protease Production

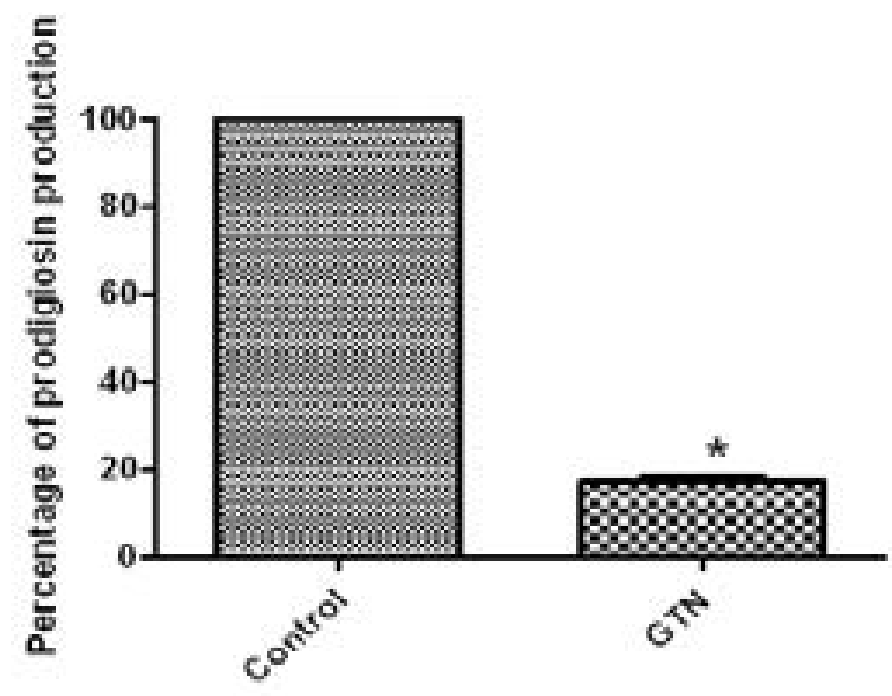

Figure 5. Inhibition of prodigiosin pigment of Serratia marcescens by GTN. *, significant $\mathbf{P}<0.05$. 
The skim milk agar method was used for the qualitative assay of protease inhibition. GTN could interfere with protease production as shown by decreasing the clear zone of proteolysis (Fig. 6).

Inhibition of swimming and swarming
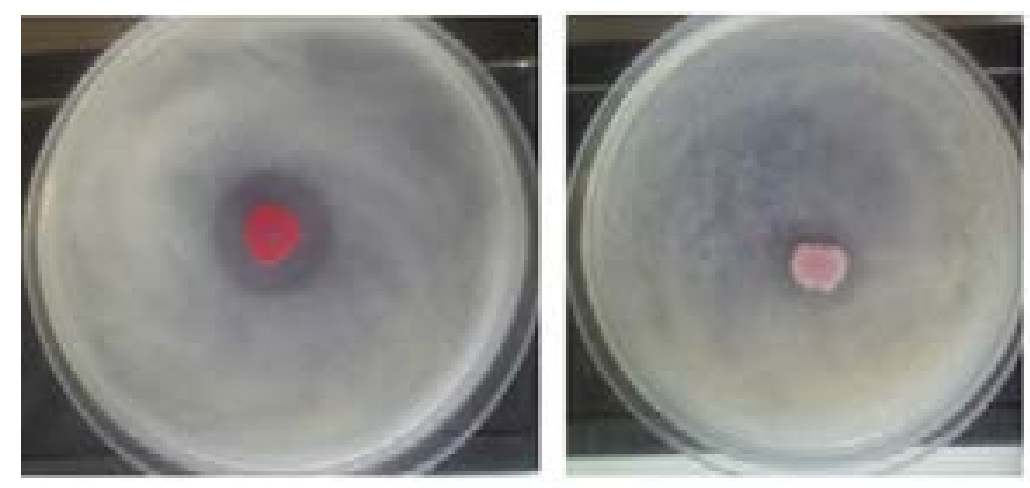

Figure 6. Inhibition of protease production by the skim milk agar method.

Swimming and swarming motility are important for ad- ing motility was decreased to the level of $79.31 \%$ (Fig. hesion and biofilm formation. In the presence of GTN, 7\&8).

swimming motility was reduced by $36.72 \%$, while swarm- Discussion
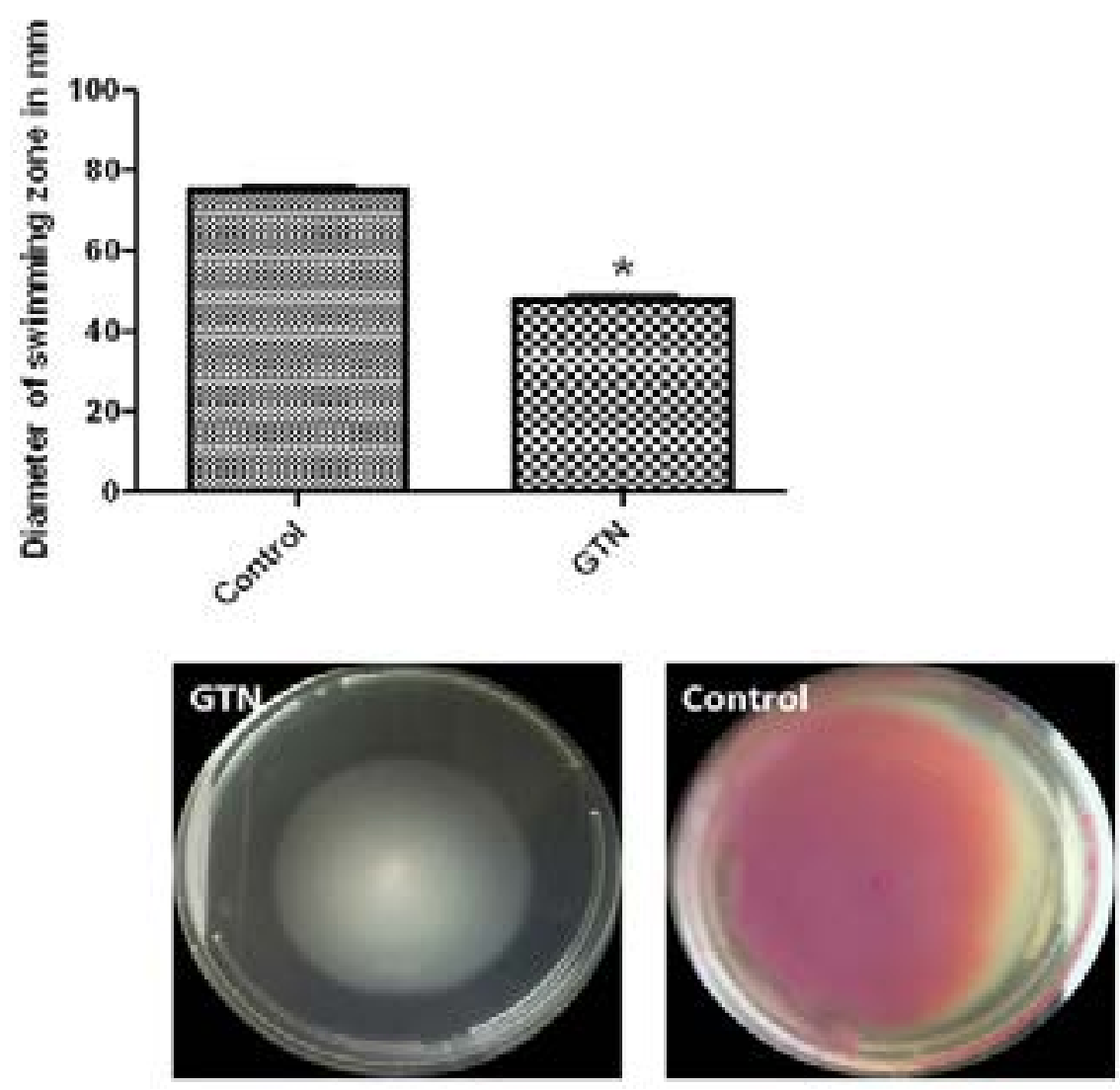

Figure 7. Inhibition of swimming motility by GTN. *, significant $\mathrm{P}<0.05$. 


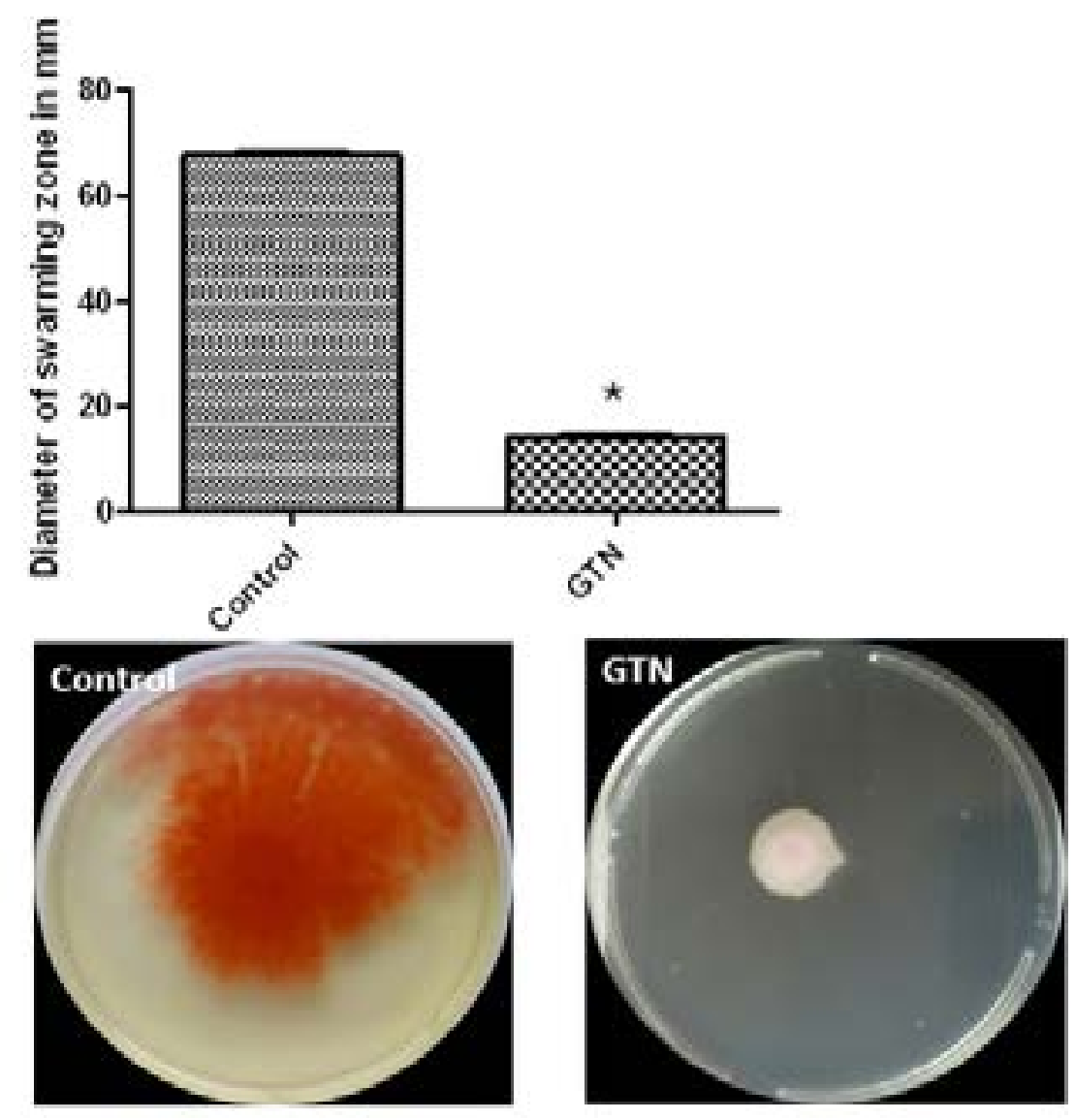

Figure 8. Inhibition of swarming motility by GTN. *, significant $P<0.05$.

Serratia marcescens is a nosocomial bacterium that is considered the seventh most common pathogen that causes nosocomial pneumonia and the tenth most common one that is responsible for hospital acquired blood stream infections ${ }^{24}$.

This study was performed to detect the possible quorumsensing mediated virulence factors inhibition by sub-inhibitory concentration of glyceryl trinitrate in Serratia marcescens.

The underlying reason for targeting quorum sensing is to bypass the stress on bacterial growth that leads to emergence of resistance and finding another way to treat infection in the light of the high antibiotic resistance of Serratia marcescens ${ }^{25}$. Quorum sensing is a key regulator of the pathogenicity in many bacteria. Inhibition of quorum sensing may be beneficial in attenuation of virulence and the treatment of infections caused by resistant strains of bacteria ${ }^{26,27}$.

The tripyrrole red prodigiosin pigment is synthesized under the control of quorum sensing in $S$. marcescens ${ }^{21}$. GTN caused remarkable reduction in prodigiosin pigment. Protease is involved in the pathogenicity of $S$. marcescens. GTN reduced the production of protease ${ }^{28}$. In this study, GTN showed high ability to inhibit biofilm. Biofilms render the infection difficult to treat due to the high resistance to antibiotics and immunity of the host ${ }^{29}$. The ability of Serratia marcescens to swim and swarm affects adhesion and biofilm formation. As a result, interference with swarming could affect biofilm production ${ }^{30,31}$. GTN blocked swarming motility.

The inhibition of virulence factors production of Serratia marcescens may be due to interference with quorum sensing. This hypothesis was proved by the ability of GTN to cause significant reduction in the production of the quorum-sensing regulated violacein pigment in the biosensor strain C. violaceum CV026. Moreover, GTN was previously reported as a quorum sensing inhibitor in Pseudomonas aeruginosa PAO1 strain ${ }^{11}$.

GTN has the advantage of FDA approval that makes 
GTN clinical application possible. This is very important because most quorum sensing inhibitors have toxic effects that hinder their use in treatment. Serratia marcescens causes surgical wounds infections and urinary tract infections. GTN can be used topically in the treatment of surgical site infections or as a catheter lock solutions for catheter associated urinary tract infections ${ }^{32}$.

\section{Conclusion}

The emergence of antibiotic resistance makes it obligatory to seek for new therapeutic options for infection control. GTN may be a useful agent in this approach because of its anti-quorum sensing and anti-virulence activities against $S$. marcescens. Targeting quorum sensing regulated virulence poses no growth pressure on bacteria and hence the resistance is much less likely to develop.

\section{Conflict of interests}

There is no conflict of interest

\section{References}

1. Hejazi A, Falkiner FR. Serratia marcescens. Journal of Medical Microbiology. 1997; 46:903-912.

2. Rice SA, Koh KS, Queck SY, Labbate M, Lam KW, Kjelleberg S. Biofilm formation and sloughing in Serratia marcescens are controlled by quorum sensing and nutrient cues. Journal of Bacteriology. 2005; 187:3477-3485.

3. Sarah JC, Neil RW, Abigail KPH, David RS, George PCS. Metabolic and regulatory engineering of Serratia marcescens: mimicking phage-mediated horizontal acquisition of antibiotic biosynthesis and quorum-sensing capacities. Microbiology. 2006; 152:1899-1911.

4. Rutherford ST, Bassler BL. Bacterial Quorum Sensing: its role in virulence and possibilties of its control. Cold Spring Harbor Prespectives in Medicine 2012; 2(11): a012427.

5. Stock I, Burak S, Sherwood KJ, Gruger T, Wiedemann B. Natural anti-microbial susceptibilities of strains of 'unusual' Serratia species: S. ficaria, S. fonticola, S. odorifera, S. plymuthica and S. rubidaea. Journal of Antimicrobial Chemotherapy. 2003; 51:865-885.

6. Traub WH. Antibiotic susceptibility of Serratia marcescens and Serratia liquefaciens. Chemotherapy. 2000; 46:315321.

7. Cegelski L.,Marshall GR, Eldridge GR, Hultgren SJ. The biology and future prospects of anti-virulence thera- pies. Nature Reviews Microbiology. 2008; 6: 17-27.

8. Rasko DA, Sperandio V. Anti-virulence strategies to combat bacteria-mediated disease. Nature Reviews Drug Discovery. 2010; 9: 117-128.

9. Gorfine SR. Treatment of benign anal disease with topical nitroglycerin. Diseases of the Colon and Rectum. 1995; 38:453-457.

10. Palmeira-de-Oliveira A, Ramos AR, Gaspar C, et al.In vitro anti-Candida activity of lidocaine and nitroglycerin: alone and combined. Infectious Diseases in Obstetrics and Gynecology. 2012; Article ID 727248, 4 pages.

11. Abbas HA, Shaldam MA. Glyceryl trinitrate is a novel inhibitor of quorum sensing in Pseudomonas aeruginosa. African Health Sciences. 2016; 16(4): 1117-1125.

12. Rosenblatt J, Reitzel RA, Raad I. Caprylic acid and glyceryl trinitrate combination for eradication of biofilm. Antimicrobial Agents and Chemotherapy. 2015; 59:1786-1788.

13. Fenton C, Wellington K, Easthope SE. 0.4\% nitroglycerin ointment: in the treatment of chronic anal fissure pain. Drugs. 2006; 66:343-349.

14. Abbas HA, Hegazy WA. Targeting the virulence factors of Serratia marcescens by ambroxol. Roumanian Archives of Microbiology and Immunology 2017; 76: In press.

15. Clinical and Laboratory Standards Institute. Methods for dilution anti-microbial susceptibility tests for bacteria that grow aerobically; Approvated standard, CLSI Document M07-A9, Vol. 2012; 32, No. 3. Wayne, PA, USA.

16. Nalca Y, Jansch L, Bredenbruch F, Geffers R, Buer J, Haussler S. Quorum-sensing antagonistic activities of azithromycin in Pseudomonas aeruginosa PAO1: a global approach. Antimicrobial Agents and Chemotherapy. 2006; 50: 1680-1688.

17. Choo JH, Rukayadi Y, Hwang JK. Inhibition of bacterial quorum sensing by vanilla extract. Letters in Applied Microbiology. 2006; 42 (6): 637-641.

18. Abraham SVPI, Palani A, Ramaswamy BR, Shunmugiah KP, Arumugam VR. Antiquorum sensing and antibiofilm potential of Capparis spinosa. Archives of Medical Research. 2011; 42, 658-668.

19. Sarkar R, Chaudhary SK, Sharma A Yadav KK, Nema NK, Sekhoacha M et al. Antibiofilm activity of Marula- A study with the standardized bark extract. Journal of Ethnopharmacology. 2014; 154 (1), 170-175.

20. Gowrishankar S, Poornima B, Pandian SK. Inhibitory efficacy of cyclo(Lleucyl-L-prolyl) from mangrove 
rhizosphere bacterium-Bacillus amyloliquefaciens (MMS-50) toward cariogenic properties of Streptococcus mutans. Research in Microbiology. 2014; 165 (4), 278-289.

21. Matsuyama T, Kaneda K, Nakagawa Y, Isa K, Hara-Hotta H, Yano I. A novel extracellular cyclic lipopeptide which promotes flagellum-dependent and -independent spreading growth of Serratia marcescens. Journal of Bacteriology. 1992; 174:1769-1776.

22. Morohoshi T, Shiono T, Takidouchi K, Kato M, Kato $\mathrm{N}$, Kato J et al. Inhibition of quorum sensing in Serratia marcescens AS-1 by synthetic analogs of $\mathrm{N}$-acyl homoserine lactone. Applied and Environmental Microbiology. 2007; 73:6339-6344

23. Huber B, Riedel K, Hentzer M, Heydorn A, Gotschlich A, Givskov Metal. The cep quorum-sensing system of Burkbolderia cepacia H111 controls biofilm formation and swarming motility. Microbiology. 2001; 147:2517-2528.

24. Jones RN. Microbial etiologies of hospital-acquired bacterial pneumonia and ventilator-associated bacterial pneumonia. Clinical Infectious Diseases. 2010; 51(1): S81S87.

25. Coulthurst SJ, Williamson NR, Harris AKP, Spring DR, Salmond GPC. Metabolic and regulatory engineering of Serratia marcescens: mimicking phage mediated horizontal acquisition of antibiotic biosynthesis and quorum-sensing capacities. Microbiology. 2006; 152, 1899_ 1911.

26. Hentzer M, Givskov M. Pharmacological inhibition of quorum sensing for the treatment of chronic bacterial infections. Journal of Clinical Investigation. 2003; 112: 1300-1307.

27. March JC, Bentley WE. Quorum sensing and bacterial cross-talk in biotechnology. Current Opinion in Biotechnology. 2004; 15: 495-502.

28. Wei JR, Lai HC. 2006. N-acylhomoserine lactone-dependent cell-to-cell communication and social behavior in the genus Serratia. International Journal of Medical Microbiology. 2006: 296, 117-124.

29. Costerton JW, Stewart PS, Greenberg EP. Bacterial biofilms: a common cause of persistent infections. Science. 199; 284, 1318-1322.

30. Shanks RMQ, Lahr RM, Stella NA, Arena KE, Brothers KM, Kwak DH et al. A Serratia marcescens PigP homolog controls prodigiosin biosynthesis, swarming motility and hemolysis and is regulated by cAMP-CRP and HexS. PLoS One. 2013; 8 (3), e57634.

31. Soo PC, Horng YT, Chang YL, Tsai WW, Jeng WY, Lu CC et al. ManA is regulated by Rss AB signaling and promotes motility in Serratia marcescens. Research in Microbiology. 2014; 165, 21-29.

32. Galloway WR, Hodgkinson JT, Bowden S, Welch M, Spring DR. Applications of small molecule activators and inhibitors of quorum sensing in Gram-negative bacteria. Trends in Microbiology. 2012; 20:449-458. 